\title{
A Nonliquid Crystal Display Screen Computer for Treatment of Photosensitivity and Computer Screen Intolerance in Post-Concussion Syndrome
}

\author{
Ann Mansur, ${ }^{1,2}$ Tyler M. Hauer,, ${ }^{1,2}$ Mohammed Wasif Hussain,,2 Mohammed K. Alatwi,,2 \\ Apameh Tarazi,, ${ }^{1,2}$ Mozhgan Khodadadi, and Charles H. Tator ${ }^{1-3}$
}

\begin{abstract}
Liquid crystal display (LCD) screens refresh at a rate of 60 times per second, which can be perceived by concussed individuals who have photosensitivity, leading to computer intolerance. A non-LCD computer screen that refreshes at a much lower rate could relieve this photosensitivity and computer screen intolerance in patients with post-concussion syndrome (PCS). Twenty-nine patients with PCS, computer intolerance, and photosensitivity performed a reading task for a maximum of $30 \mathrm{~min}$, with an LCD computer or a non-LCD device, and were given a comprehension test after completion of the reading task. The Sport Concussion Assessment Tool 3 was administered before and after each reading task. Symptom scores, amount of time spent reading, and performance on the comprehension tests were compared between the two devices. Patients also completed a self-report questionnaire of their subjective experience. The LCD screen computer produced significantly greater symptom exacerbation (median difference $=5, \mathrm{~W}=315, p<0.01$ ) and a greater number of symptoms (median difference $=1, \mathrm{~W}=148, p<0.01$ ) than the non-LCD screens. The non-LCD screen resulted in a longer symptom-free reading time (median $=48 \mathrm{sec}, \mathrm{W}=147, p<0.01$ ), but not a greater number of words read (median $=281, \mathrm{~W}=148, p=0.098)$. Females were more likely to have greater symptom exacerbation with the LCD screen $(U=14.0, p<0.01)$. No significant difference was found in performance on the comprehension test. Subjective reports showed that the non-LCD experience was more favorable, and most patients stated they would recommend this device for other patients with PCS. This study is the first to show the clinical utility of non-LCD screen computers in the management of photosensitivity and computer screen intolerance in patients with PCS. The non-LCD screen computer has the potential to facilitate return-to-work or return-to-school in concussed individuals.
\end{abstract}

Keywords: computer screen intolerance; photosensitivity; post-concussion syndrome; traumatic brain injury

\section{Introduction}

$\mathbf{C}$ ONCUSSION is the most common type of traumatic brain injury (TBI) and is defined by the International Consensus Conference on Concussion in Sports as "a complex pathophysiological process affecting the brain, induced by bio-mechanical forces." 1 Most patients will recover within 28 days; however, at least 10 $15 \%$ of patients will have post-concussion syndrome (PCS), where symptoms can persist for months to years. ${ }^{2}$ PCS is marked by a constellation of physical, cognitive, and emotional symptoms, including photosensitivity or light sensitivity. Indeed, light sensitivity can persist for weeks, months, or years after various types of brain injury, ${ }^{3-5}$ and has been shown to be one of the few early symptoms predictive of the future development of PCS. ${ }^{6}$ Previous studies on management options of photosensitivity in concussed individuals suggest that the neural adaptation of photosensitivity is a long-term process. ${ }^{5}$

Photosensitivity is highly debilitating because it directly impairs day-to-day functioning, including the use of computer screens and hand-held communication devices. Computers refresh at $60 \mathrm{~Hz}$, which makes it difficult for patients with PCS to fuse images at that flicker frequency. Most PCS patients with photosensitivity also have difficulty with computer screens, for which we have coined the term computer screen intolerance, a unique post-concussion symptom involving discomfort with screens that refresh at high frequency, including cell phones and computers.

\footnotetext{
${ }^{1}$ Faculty of Medicine, University of Toronto, Toronto, Ontario, Canada.

${ }^{2}$ Canadian Concussion Centre, Toronto, Ontario, Canada.

${ }^{3}$ Division of Neurosurgery, Toronto Western Hospital, Toronto, Ontario, Canada.
}

(C) Ann Mansur et al., 2018; Published by Mary Ann Liebert, Inc. This Open Access article is distributed under the terms of the Creative Commons Attribution Noncommercial License (http://creativecommons.org/licenses/by-nc/4.0/) which permits any noncommercial use, distribution, and reproduction in any medium, provided the original author(s) and the source are credited. 
Currently, there are very few evidence-based treatments for PCS. ${ }^{2}$ Physicians generally advise avoidance of activities that enhance symptoms, including photosensitivity or computer screen intolerance; hence, patients are advised to avoid using liquid crystal display (LCD) screens because they can exacerbate symptoms and interfere with recovery. ${ }^{2}$ Computers are a widely used platform for daily communication, however, and are often a required technology in workplaces and schools. Finding ways to improve computer screen tolerance in these populations will not only prevent social isolation, but may facilitate earlier return to work or school, and potentially a faster recovery.

As noted above, LCD computer screens refresh or flicker at a rate of 60 times per second. This is tolerable for a healthy individual, but studies have shown that concussed individuals with photosensitivity have a higher critical flicker frequency (CFF) threshold. ${ }^{7,8}$ This means that patients with concussions may perceive light as flickering at higher flicker frequencies, leading to headaches, cognitive fatigue, and eyestrain. ${ }^{8}$ Flashing and motion of light can produce nausea during recovery from a concussion and thus can delay recovery. ${ }^{9}$ The backlighting of the LCD computer screen can also stress individuals who experience computer screen intolerance and can cause cognitive fatigue, headache, and eye fatigue. ${ }^{10}$ While it is possible to mitigate some of the eyestrain caused by LCD screens by modifying the brightness, glare, and color transmission, none of these solutions change the flicker frequency of computer screens.

A new non-LCD computer screen has been designed by Iris Technologies, a company in Toronto, Canada. Their technology displays images on a magnetized polymer screen, as opposed to light modulating liquid crystals on an LCD screen. This screen has no backlighting and does not flicker; instead, it uses ambient lighting to illuminate the screen and refreshes only when the content of the page is changed.

The purpose of this study was to assess the feasibility of a nonLCD screen to mitigate computer screen intolerance in patients with PCS. We hypothesized that patients with PCS would be able to read word passages with less symptom exacerbation and for a longer period using a non-LCD screen computer than a LCD screen computer, and that their reading comprehension would be better with the non-LCD screen.

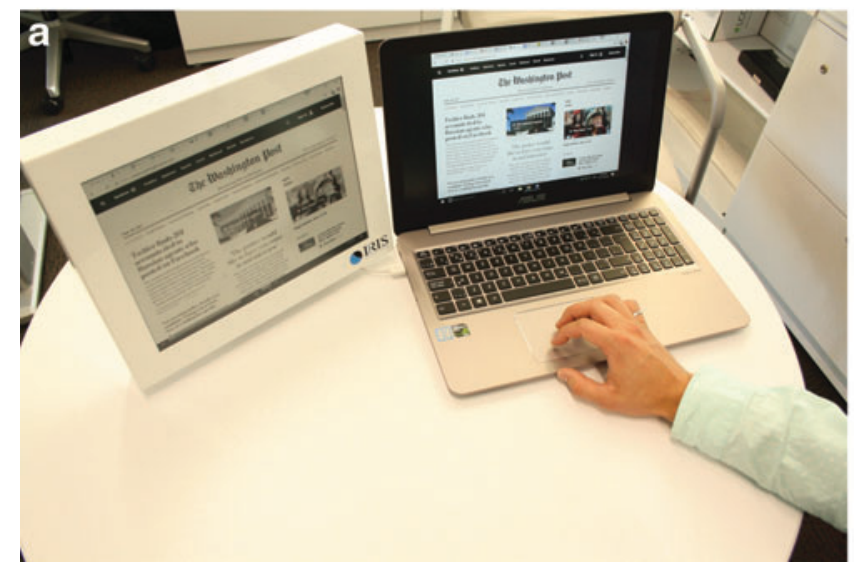

\section{Methods}

This study was conducted at the Canadian Concussion Centre, Toronto Western Hospital in Toronto, Canada, and was approved by the Research Ethics Board at the University Health Network, which includes the Toronto Western Hospital. All participants provided written informed consent before their inclusion in the study.

\section{Participants}

Adult patients were recruited from the Concussion Clinics or the Concussion Education and Support Group of the Canadian Concussion Centre, Toronto Western Hospital. Eligible patients were (1) between the ages of 16 and 80, (2) had a diagnosis of PCS as per the International Classification of Disease 10.0 criteria, (3) sustained the concussion greater than three months before the date of testing, (4) endorsed difficulties in tolerating computer screens (which we term computer screen intolerance), and (5) were proficient in English. Patients were ineligible if they did not have PCS, had a brain injury more severe than a concussion, or had another neurological, ocular, or psychiatric condition. Eligible participants were identified by the senior author (CHT).

\section{Device}

Two computer devices were used in this study: a 15.0" LCD personal computer screen and a similar sized non-LCD computer (first generation) designed by Iris Technologies. This non-LCD screen is a separate monitor that attaches by USB to the LCD screen and mirrors the contents that are on the LCD display (Fig. 1). It uses magnetized polymers to create shapes, as opposed to the light modulating liquid crystals on an LCD screen. This non-LCD screen is nonbacklit and does not constantly refresh the page the way an LCD screen flickers; instead, it refreshes only when the content on the screen is changed, and it refreshes at only 8-12 frames per second. This technology is similar to commercialized hand-held devices including the Kindle and Kobo e-readers.

The resolution of the LCD screen was reduced to $1600 \times 1200$ so that the effective screen area for both screens was the same. The screens differ in contrast; contrast refers to the difference between the darkest dark pixel and the brightest white pixel. It is expressed as a ratio. The contrast of a typical modern LCD screen is 1000:1, whereas the contrast was 12:1 in the non-LCD screen. Thus, the

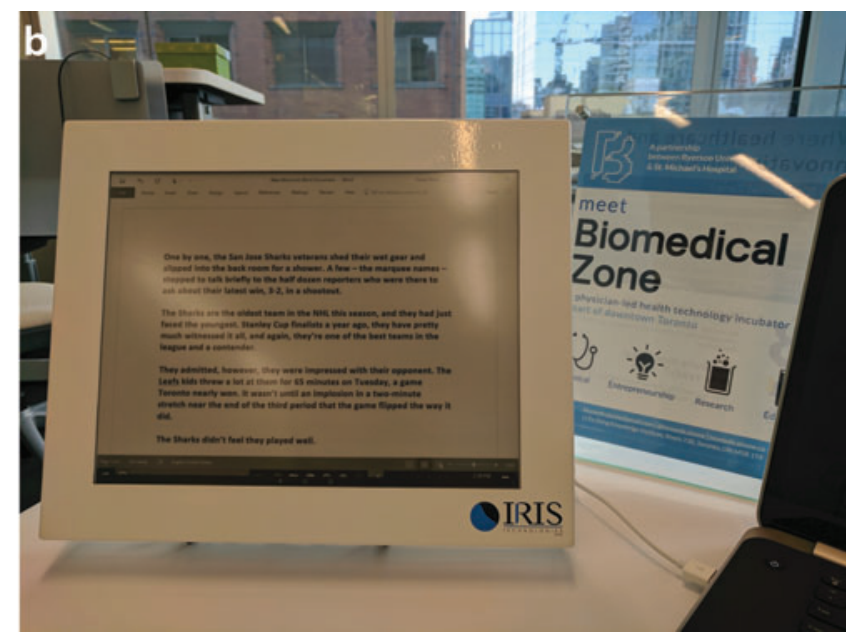

FIG. 1. The study devices. (a) The non-liquid crystal display (LCD) device on the left and LCD device on the right demonstrating webpage data; (b) the non-LCD device on the left and the LCD device on the right demonstrating a reading task. 
non-LCD screen has reduced contrast in comparison with the LCD screen, but has greater contrast than print media.

\section{Procedures}

An outline of the study protocol is illustrated in Figure 2. Participants were required to make two visits, at least $24 \mathrm{~h}$ apart. On each visit, they were asked to complete a silent reading task on one of the devices until any of their PCS symptoms were exacerbated, but only for a maximum of $30 \mathrm{~min}$. The order of the devices was randomized. The reading excerpt was designed by the study team using nonlicensed online material chosen from material written at the Grade 5

Patients in CCC Concussion Clinic, $\mathrm{n}=29$

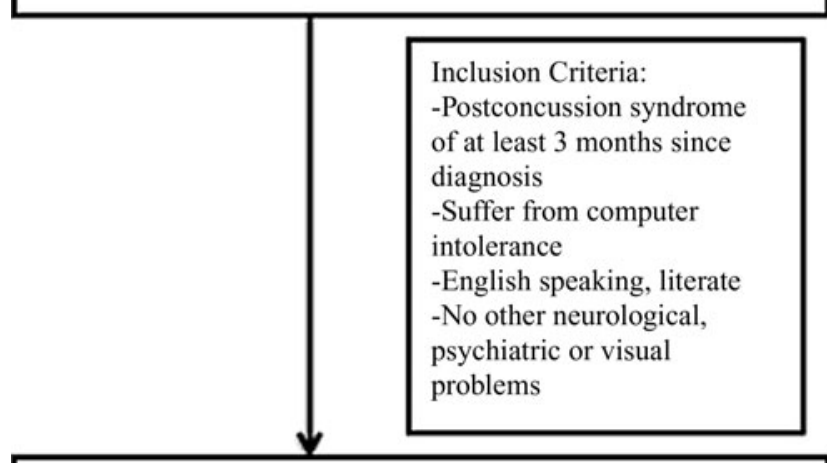

Baseline data: demographics, clinical history, SCAT3

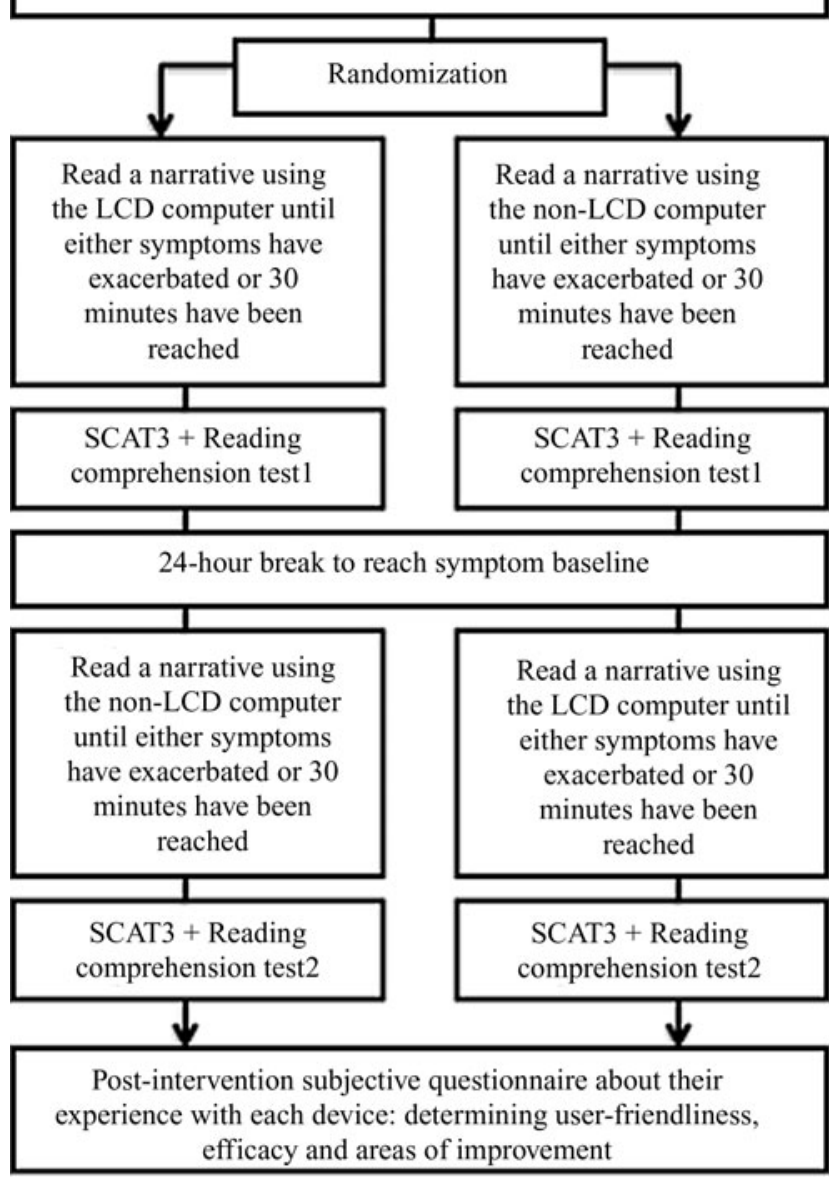

FIG. 2. Summary of study protocol. CCC, Canadian Concussion Centre.
English level, at which all participants would be proficient. ${ }^{11}$ The excerpts were not from popular stories that participants may have been exposed to in the past. The content of the two reading tasks, one for each device, was different to avoid any practice effects.

The symptom component of the Sports Concussion Assessment Tool 3 (SCAT3) was completed before and after the reading task to quantify the change in symptom number and severity attributed to the reading task. The SCAT3 is a validated clinical assessment protocol that lists 22 symptoms of concussion with a severity scale of 0-6 for each, and with a maximum score of 132; it is used widely in concussion assessment but does not yet have published normative data (SCAT3, http://links.lww.com/JSM/A30). ${ }^{1}$

After completion of the reading task, participants were asked to complete a comprehension test of the material they read, but were only required to answer questions pertaining to the information they were able to read. At the end of the second visit, participants completed a self-reported questionnaire of their experience with the two devices. The questionnaire included both multiple choice and short answer feedback (Appendix A). Both interventions occurred in the same room, which had two incandescent light sources for background illumination; the latter is important because the LCD screen diminishes its brightness in the presence of a bright environment whereas the non-LCD screen reflects the ambient light. Therefore, we aimed to keep the effects of ambient lighting consistent between participants and study visits. Lastly, the devices were kept at a standard distance and inclination from the participant's seat.

The study personnel involved in administering the tasks were blinded to the patients' clinical information. Different members of the team conducted the data analysis to avoid any bias.

\section{Data analysis}

Variables assessed:

1. Symptoms: the symptom severity for each symptom listed in the SCAT3, the change in total symptom severity after the reading task, and the change in the number of symptoms endorsed after the reading task.

2. Reading: the number of words read, and the time spent reading.

3. Comprehension: the number of questions answered, and the percentage of questions answered correctly.

4. Subjective experience: experience with the non-LCD screen using a Likert scale, aspects they liked about the non-LCD screen and areas of improvement, subjective symptom change with each device, and the commercial potential of the non-LCD device. These topics were addressed using a combination of Likert scale, multiple choice. and shortanswer questions.

The change in symptom severity, change in number of symptoms, and change in specific symptoms with the LCD and non-LCD device were analyzed using the Wilcoxon Signed Rank Test. The reading time, number of words read, number of comprehension questions answered, number of comprehension questions answered correctly, and percent of comprehension questions answered correctly were analyzed by comparing the LCD screen directly to the non-LCD screen using the Wilcoxon Matched-Pairs Signed Rank Test. The change in number of symptoms and change in symptom severity were also analyzed by comparing the LCD screen directly to the nonLCD screen using the Wilcoxon Matched-Pairs Signed Rank Test.

The effect of gender on these outcome variables was assessed using a nonparametric Mann-Whitney $U$ test whereas the implication of age, number of concussions, and time since diagnosis were assessed using Spearman correlations. The sample size was 29 subjects who varied in their clinical and demographic characteristics. Our goal was to determine the clinical utility of the non-LCD screen as a 
Table 1. Demographic and Clinical Characteristics of Participants $(\mathrm{N}=29)$

\section{Participants}

\begin{tabular}{lc}
\hline Age & $40.5(14.8)$ \\
Female $(\mathrm{N}, \%)$ & $23(79.3)$ \\
Time since diagnosis (years) & $1.3(1.1)$ \\
Number of concussions & $2.6(1.8)$ \\
Photophobia $(\mathrm{N}, \%)$ & $29(100.0)$
\end{tabular}

All data are reported as mean (standard deviation) unless otherwise specified.

treatment option for screen intolerance, and therefore we were conservative with the statistical analysis and reported outcomes as significant at $p<0.01$.

Qualitative data were analyzed using grounded theory, whereby data were coded to devise common themes that inform theories.

\section{Results}

\section{Quantitative results}

Table 1 summarizes the demographic and clinical characteristics of the 29 participants.

The average age was 40.5 , with a range from 16-67. Twenty-three participants $(79.3 \%$ ) were female. The average time since diagnosis was 1.3 years, and the average number of concussions sustained was 2.6.

A summary of the reading outcomes for the LCD screen computer, the non-LCD screen computer, and the two compared is provided in Table 2a, b, and c, respectively. Reading on the LCD screen caused a significant increase in the total SCAT3 score, by an average of 12.5 points $(\mathrm{W}=390, p<0.01)$ and produced an average of two new symptoms $(\mathrm{W}=257, p<0.01)$. In contrast, reading on the non-
LCD screen did not significantly exacerbate the total symptom severity $(\mathrm{W}=138, p=0.063)$ and did not change the number of symptoms reported ( $\mathrm{W}=41, p=0.49)$. Both devices caused a feeling of pressure in the head and difficulty concentrating, but only the LCD screen resulted in worsening of additional symptoms including headache (W=178, $p<0.01$ ), feeling "in a fog" ( $\mathrm{W}=120, p<0.01)$, nausea/vomiting (W=66, $p<0.01$ ), "don't feel right" ( $\mathrm{W}=105$, $p<0.01$ ), and most notably, light sensitivity ( $\mathrm{W}=55, p<0.01$ ).

When comparing the two devices, the LCD screen resulted in significantly greater symptom exacerbation $(\mathrm{W}=315, p<0.01)$ and produced a greater number of symptoms $(\mathrm{W}=148, p<0.01)$ (Table 2c). Participants were able to tolerate the non-LCD screen for a longer period ( $\mathrm{W}=147, p<0.01$ ), but the number of words read did not differ between the two devices ( $\mathrm{W}=148, p=0.098$ ). There was also no significant difference between the two devices in terms of the number of questions answered $(\mathrm{W}=95, p=0.26)$ and the percentage of questions answered correctly ( $\mathrm{W}=25, p=0.77)$.

Females had significantly worse symptom severity than males with the LCD screen $(\mathrm{U}=22.5, p<0.01)$ and had a greater reduction in symptom severity between the two devices than their male counterparts $(\mathrm{U}=14.0, p<0.01)$. Age, number of concussions, and time since diagnosis did not cause significant changes in any outcome measure.

\section{Qualitative results}

Subjective responses to the post-intervention questionnaire are reported in Table 3. The non-LCD screen was better than average in terms of user-friendliness, ease of use, comfort, applicability, screen quality, esthetic display, and overall satisfaction (mean score $>2.5$ on a five-point Likert scale from "not very much" to "very much"). Similar to the objective findings, the non-LCD screen minimally exacerbated symptoms or was minimally bothersome to the eyes.

Table 2. Reading Task Outcomes with the Liquid Crystal Display and Non-Liquid Crystal Display Screens

A. Wilcoxon Signed Rank Test for the Liquid Crystal Display Screen Performance

\begin{tabular}{|c|c|c|c|c|c|}
\hline Outcome & $\begin{array}{l}\text { LCD screen } \\
\text { (Pre) }\end{array}$ & $\begin{array}{c}\text { LCD screen } \\
\text { (Post) }\end{array}$ & $\begin{array}{c}\text { LCD screen } \\
\text { (delta) }\end{array}$ & $\begin{array}{l}\text { Sum of signed } \\
\text { ranks }(W)\end{array}$ & $\mathrm{p}$ value \\
\hline Total symptom severity (SCAT3 total symptom score) & $30.2(25.8)$ & $42.7(20.1)$ & $12.5(2.5)$ & 390 & $<0.01$ \\
\hline Number of SCAT3 symptoms produced & $12.1(6.3)$ & $14.1(6.5)$ & $2.0(0.5)$ & 257 & $<0.01$ \\
\hline
\end{tabular}

B. Wilcoxon Signed Rank Test for the non- Liquid Crystal Display Screen Performance

\begin{tabular}{|c|c|c|c|c|c|}
\hline Outcome & $\begin{array}{c}\text { non-LCD } \\
\text { screen (pre) }\end{array}$ & $\begin{array}{c}\text { non-LCD } \\
\text { screen (post) }\end{array}$ & $\begin{array}{c}\text { non-LCD } \\
\text { screen (delta) }\end{array}$ & $\begin{array}{l}\text { Sum of signed } \\
\text { ranks }(W)\end{array}$ & $\mathrm{p}$ value \\
\hline Total symptom severity (SCAT3 total symptom score) & $30.0(21.1)$ & $33.1(21.0)$ & $3.0(1.5)$ & 138 & 0.063 \\
\hline Number of SCAT3 symptoms produced & $12.9(5.8)$ & $13.2(5.9)$ & $0.3(0.4)$ & 41 & 0.49 \\
\hline
\end{tabular}

C. Wilcoxon Matched-Pairs Signed Rank Test Comparing the Two Screens

\begin{tabular}{|c|c|c|c|c|c|}
\hline Outcome & $\begin{array}{c}L C D \text { minus } \\
\text { non- } L C D \text { (pre) }\end{array}$ & $\begin{array}{c}L C D \text { minus } \\
\text { non-LCD (post) }\end{array}$ & $\begin{array}{c}L C D \text { minus } \\
\text { non- } L C D(\text { delta })\end{array}$ & $\begin{array}{l}\text { Sum of Signed } \\
\text { Ranks }(W)\end{array}$ & $\mathrm{p}$ value \\
\hline Total symptom severity (SCAT3 total symptom score) & $0.2(24.3)$ & $9.6(20.3)$ & 5 & 315 & $<0.01$ \\
\hline Number of SCAT3 symptoms produced & $-0.8(5.9)$ & $0.9(6.2)$ & 1 & 148 & $<0.01$ \\
\hline Number of questions answered correctly & & & 1 & 95 & 0.26 \\
\hline Number of questions answered & & & 3 & 127 & 0.11 \\
\hline$\%$ of questions answered correctly & & & 0.5 & 25 & 0.77 \\
\hline Number of words read & & & 261 & 146 & 0.098 \\
\hline Time of reading (seconds) & & & -48 & 147 & 0.009 \\
\hline
\end{tabular}

LCD, liquid crystal display; SCAT3, Sports Concussion Assessment Tool 3. 
Table 3. Subjective Responses to Post-Intervention QUESTIONNAIRE

A. Comparison of the Two Devices

\begin{tabular}{lc}
\hline Characteristic of non-LCD device & Response (mean, SD) \\
\hline User-friendliness & $3.9(0.9)$ \\
Easy to use & $4.2(0.8)$ \\
Comfortable to use & $4.1(0.6)$ \\
Applicable to your tasks & $4.1(0.9)$ \\
Exacerbate symptoms & $2.1(1.0)$ \\
Bothers your eyes & $1.7(0.8)$ \\
Screen quality & $3.5(1.0)$ \\
Esthetic display & $3.3(1.1)$ \\
Overall satisfaction & $4.0(0.7)$ \\
\hline
\end{tabular}

B. Responses to Questions about the Experience With the Non-LCD DeVICE, USING a Likert Scale

\begin{tabular}{lccc}
\hline $\begin{array}{l}\text { non-LCD computer versus } \\
\text { LCD computer }\end{array}$ & $\begin{array}{c}\text { Same } \\
(\mathrm{N}, \%)\end{array}$ & $\begin{array}{c}\text { More } \\
(\mathrm{N}, \%)\end{array}$ & $\begin{array}{c}\text { Less } \\
(\mathrm{N}, \%)\end{array}$ \\
\hline $\begin{array}{l}\text { Symptom severity } \\
\text { Length of time reading }\end{array}$ & $5(17.9)$ & $1(3.6)$ & $22(78.6)$ \\
& $8(28.6)$ & $19(67.9)$ & $1(3.6)$ \\
\cline { 2 - 4 } & $\begin{array}{c}\text { Same } \\
(\mathrm{N}, \%)\end{array}$ & $\begin{array}{c}\text { Better } \\
(\mathrm{N}, \%)\end{array}$ & $\begin{array}{l}\text { Worse } \\
(\mathrm{N}, \%)\end{array}$ \\
\cline { 2 - 4 } Comprehension of text & $14(50.0)$ & $12(42.9)$ & $2(7.1)$ \\
User-friendliness & $19(67.9)$ & $3(10.7)$ & $6(21.4)$ \\
Comfortable to use & $12(42.9)$ & $16(57.1)$ & $0(0.0)$ \\
\hline
\end{tabular}

C. Perceptions on the Commercial Potential of the Non-Liquid Crystal Display Device

\begin{tabular}{lc}
\hline Characteristic of non-LCD device $^{\mathrm{b}}$ & Response $(\mathrm{N}, \%)^{\mathrm{b}}$. \\
\hline Useful for PCS & $25(100.0)$ \\
Useful in workplaces & $26(100.0)$ \\
Would you buy a non-LCD computer? & $24(92.3)$ \\
Would you recommend a non-LCD & $27(100.0)$ \\
$\quad$ computer to others with PCS? & \\
\hline
\end{tabular}

LCD, liquid crystal display; PCS, post-concussion syndrome.

${ }^{\mathrm{a}}$ Twenty-eight responses for this set of questions.

${ }^{\mathrm{b}}$ Not all participants reported answers to these questions.

When comparing the non-LCD screen directly with the LCD screen, $78.6 \%$ of respondents thought that the non-LCD device was better in terms of less symptom exacerbation; $67.9 \%$ of respondents thought that they were able to read longer with the non-LCD screen; $21.4 \%$ of participants thought that the non-LCD screen was less user-friendly than the LCD screen, but $67.9 \%$ thought the two devices were comparable in this regard.

Analysis of the short-answer responses yielded three themes that describe the patients' perceived benefits of the non-LCD screen and areas for its improvement. Participants indicated that the reading experience with the non-LCD screen was easier on the eyes, allowed them to focus their attention better, and was an overall smoother reading experience; however, they wanted to be able to scroll through the material more easily on the non-LCD screen computer and be able to use a single monitor as opposed to have images from the LCD computer mirrored onto the second device.

The non-LCD screen resulted in a more comfortable reading experience that was easy on the eyes. Participants found that the non-LCD screen had a dimmer background, the words appeared softer to the eyes, and the reading experience was more comparable to reading print than a LCD screen. Overall, they found that these features helped them focus their attention and contributed to a less symptom-exacerbating reading experience. The specific comments included the following:

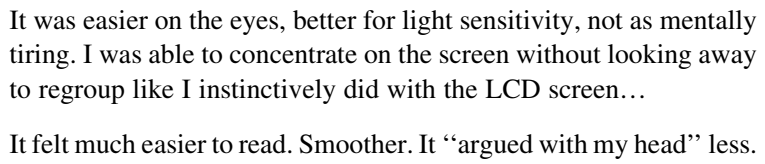

It felt much easier to read. Smoother. It "argued with my head" less.

The non-LCD screen did not quickly refresh the content on the display. Most participants thought that there was a lag in time when trying to refresh the contents on the non-LCD display. This was perceived as a pause, and not a jitter or flicker. They wanted to be able to scroll naturally through the material, the way they would with a LCD screen. Overall, the participants thought that this technical shortcoming slowed their reading and contributed to some frustration. To mitigate this shortcoming would necessitate elimination of the lag time in mirroring the content on the display, and this is under consideration.

The refresh button was too slow (moving to the next page or scrolling up and down) and this slowed me down.

Participants preferred to use a single device as opposed to navigating two screens. As mentioned previously, the nonLCD monitor is attached to a LCD monitor using a USB terminal, and the contents of the LCD monitor are mirrored onto the nonLCD display (Fig. 1). Participants thought that it was unnatural and bothersome to have to use two displays when navigating the nonLCD screen. They did not like the necessity to use the scroll function on the LCD computer while viewing the non-LCD screen. Participants would prefer the non-LCD screen computer to be a stand-alone device, or to have had the non-LCD screen mounted on the LCD computer. This would have resulted in a more userfriendly experience and would have required less desk space.

\begin{abstract}
It is counter-intuitive to reach over to the other device to go up and down. Instead, there should be readily available controls right on the non-LCD screen computer.
\end{abstract}

Have the mouse directly attached to the non-LCD screen computer to allow scrolling..

I suggest that non-LCD screen should be attachable to your existing computer...it takes brain energy to learn a new program/way of working.

The question about important factors when buying a screen yielded three themes: cost; compatibility with other computer software and devices; and wider technological applicability.

A non-LCD screen would be marketable if it was available at a reasonable cost. Participants thought it should be priced similarly to other computer devices, and that this would not only help patients buy the product, but would be a financially feasible workplace accommodation.

The non-LCD screen must be compatible with other devices and software. Respondents thought that when purchasing a computer, compatibility is needed with other devices such as a cell phone or handheld devices, computer software, and gaming systems. 
It would need to operate similarly to my current computer (i.e., be compatible).

The non-LCD screen needs to expand its technological applicability. Patients were critical of only being able to test the device with reading, and not with some of the many other applications available with computers. They thought that to be successful commercially, the device would have to be applicable to a wider variety of uses including navigating the Internet, being able to view color images, and using screenwriting programs.

...application to ALL tech users-for all functions of a regular

computer, not just reading.

What do images look like? Could it show color?

All respondents thought that the non-LCD screen would be useful for patients with PCS and would be a useful form of workplace accommodation. All patients would recommend this device to others with PCS and more than $90 \%$ would buy this product.

\section{Discussion}

To our knowledge, this is the first study to assess the use of a non-LCD screen as a therapeutic device for patients with concussion. The study showed that reading with an LCD screen resulted in a greater increase in total symptom severity and even created new symptoms, as opposed to the non-LCD screen. The non-LCD screen enabled a less symptom-exacerbating reading experience, which may make it a useful therapeutic agent for patients with PCS, photosensitivity, and computer screen intolerance. This finding was also supported by subjective responses from patients who reported an overall satisfaction with the non-LCD product, particularly because it was less symptom-provoking. Many patients thought the symptom benefit was great enough to purchase a non-LCD device and to recommend it to other patients with PCS.

Patients spent a significantly longer time reading using the nonLCD screen than the LCD device. While this might suggest that patients were able to tolerate the screen for a longer period, the finding that there was no difference in the number of words read between the two devices makes this explanation unlikely. The nonLCD screen had a pause each time the page was changed, and patients indeed criticized this pause. It is likely that the pause contributed to the additional time spent reading material on the non-LCD screen.. While this did not increase the patients' symptomatology or hinder reading comprehension, the designers of the device should work to eliminate it to optimize the non-LCD screen for patients with PCS.

The study had a larger female representation because more females volunteered for the study. Females are more likely to experience concussions and migraines, both of which include photosensitivity, and therefore, females may be more prone to experiencing computer screen intolerance. We found that the impact of the LCD screen on symptom severity and the change in symptom exacerbation between the devices was greater for females than males.

A number of concussion studies have also shown gender differences. The National Collegiate Athletic Association Injury Surveillance System from 2009 to 2014 showed that female athletes experience a greater number of concussions than males. ${ }^{12}$ Other studies have shown that females tend to report a greater number of symptoms and have a greater likelihood of PCS developing ${ }^{13}$ but that gender in itself is not a predictor of recovery after PCS. ${ }^{14}$ The possible reasons for these gender differences include differences in body structure, musculature, and organization of the brain. For example, females have lower cortical neuronal densities and higher neuropil numbers. ${ }^{15-17}$ Females have a greater basal rate of glucose metabolism ${ }^{17,18}$ and different ionic cascades that can lead to worse outcomes. ${ }^{18,19}$ Females also have a greater incidence of depression after mTBI. ${ }^{17}$

Current research on screen intolerance in concussion has focused primarily on photosensitivity and therapeutics that address its biological basis. ${ }^{20}$ One study of functional magnetic resonance imaging in patients with concussions found increased connectivity in the right frontoparietal network. The authors suggest that patients with brain injuries might have photosensitivity because they have a heightened awareness of visual stimuli in comparison with healthy controls, which is based on increased connectivity in the regions of the brain responsible for sensory perception. ${ }^{21}$ Hence, one theory is that photosensitivity is mediated by increased awareness of light stimuli.

Other studies attributed photosensitivity to visual dysfunction in concussion. Research on blast injuries (a type of mTBI) has shown that soldiers with these injuries have a variety of oculomotor deficits in accommodation, version, reading speed, and reading comprehension in comparison with healthy controls. ${ }^{22}$ The direct implications of accommodative dysfunction on photosensitivity has been explored further by Truong and Ciuffreda ${ }^{23}$ who compared the pupillary light reflex and twelve pupil parameters in healthy participants with photosensitivity and concussed individuals with photosensitivity. They found that these populations differ in their pupillary responses to light stimuli, and that pupillary changes can be a valuable biomarker of photosensitivity. ${ }^{23}$

Other patients may also have heterophoria, which is the misalignment of the eyes; consequently, they rely excessively on certain extraocular muscles, thereby contributing to eyestrain. While this does not directly relate to photosensitivity, the eyestrain can contribute to a subjective photosensitive experience. Prism glasses have been proposed for the latter, although there is limited research to support this therapy. ${ }^{24}$

Several studies have investigated the therapeutic value of chromatic lenses or filters in concussion. The concept behind these devices is that blue light is between the 400 to $450 \mathrm{~nm}$ wavelength and activates the $\mathrm{S}$ cones in the retina; given the flatter and wider midpoint of this focal area, blue light is focused myopically as opposed to red light. Filtering out the blue light decreases accommodative demand and overall eyestrain. Filters also decrease brightness, which is thought to contribute to photosensitivity. In fact, an older study by Waddell and Gronwall ${ }^{3}$ argued that brightness is a direct measure of photosensitivity.

Noseda and colleagues ${ }^{25}$ used a rat model to explore the anatomic basis of photosensitivity in migraine headaches. They found that there are intrinsically photosensitive retinal ganglion cells that express melanopsin, which acts as a photoreceptor and irradiance detector; these cells are innervated by the trigeminovascular pathway and project directly to the pain centers in the posterior thalamus, thereby mediating the pain response to illuminance (energy of light emitted per second).

Various commercial software such as f.lux (f.lux, https:// justgetflux.com) can alter both the spectral composition and luminance of computer screens. Research on chromatic filters and glasses in patients with concussion yielded inconclusive results; although patients may endorse some relief with these technologies, ${ }^{26}$ the results on objective visual performance are nonsignificant. ${ }^{27-33}$

Alteration of luminance and spectral composition of screens have been proposed as therapeutic strategies for a variety of 
populations that experience photosensitivity. For example, chromatic glasses have been proposed for patients with nonepileptic photosensitivity ("photoparoxymsal responsiveness"), ${ }^{34}$ visually sensitive migraines, ${ }^{35}$ acquired brain injury, ${ }^{28}$ and stroke. ${ }^{27}$ Computer glasses are glasses with an anti-glare film that are marketed for various conditions and have also been used in healthy adults with eyestrain from excessive use of computer screens, termed computer vision syndrome. ${ }^{35}$ None of the recommendations, however, are based on validated empirical data and, importantly, they do not address the flickering associated with LCD screens.

The present study represents the first attempt to provide a therapeutic device for LCD screen intolerance in PCS by addressing the change in CFF that occurs in this disease in addition to brightness; however, there are some methodological limitations. Although our patients all had PCS and screen intolerance, there was heterogeneity in terms of time since concussion, number of concussions, and methods of injury. Studies with a larger clinical sample are needed to replicate these findings and to investigate the role of these demographic and clinical factors on the therapeutic value of non-LCD screens in PCS.

Second, the devices differed in light qualities such as contrast ratio and their manipulation of ambient light in addition to flicker frequency; while we could not control for the difference in contrast ratios, we tried to control for further changes in contrast by keeping the source of ambient light consistent among study sessions and participants. In addition, this study only explored the feasibility of the non-LCD screen on reading; other computer functions such as navigating the Internet, writing, and multi-tasking were not assessed.

Last, this study focused on self-reported symptoms with SCAT3 as a measure of computer intolerance. Other potential measures of computer intolerance such as PCS-specific questionnaires including the Post-Concussion Symptom Inventory and Post-Concussion Symptom Scale, ${ }^{36}$ oculomotor tracking, pupillary response, ${ }^{23,37}$ and brightness measures could have been explored for a more comprehensive assessment of the pathophysiology of computer intolerance in PCS. Also, specific studies of the non-LCD computer screen should be performed on return to learn in children with PCS and on return to work in adults with PCS.

\section{Conclusions}

This study is the first clinical research on the use of a non-LCD device to address the issue of computer screen intolerance in PCS. Our study is a step toward treating screen intolerance in PCS. Future studies are needed with larger samples and in select populations, including broader age groups, and with specific return to school and return to work populations. In addition, studies should be performed on other neurological conditions in which photosensitivity is a factor such as migraines or epilepsy. In an everincreasing digital era, computer screens are necessary tools to function in society. Hence, future work in the field of computer screen intolerance and concussion is paramount and has valuable implications on return-to-work, return-to-school, and ultimately, return to function.

\section{Acknowledgments}

We would like to thank Iris Technologies for providing the nonLCD screen for the study, and for specially configuring it to our specifications for use with PCS patients. Thank you to Catherine Wiseman-Hacks for her support in developing the reading task.

This study was funded by the Ontario Brain Institute and the Toronto General and Western Hospital Foundation. Iris Technol- ogies provided the non-LCD screen computer, but did not contribute funding to the study.

\section{Author Disclosure Statement}

No competing financial interests exist.

\section{References}

1. McCrory, P., Meeuwisse, W., Dvorak, J., Aubry, M., Bailes, J., Broglio, S., Cantu R.C., Cassidy, D., Echemendia, R.J., Castellani, R.J., Ellenbogen, R., Emery, C., Engebretsen, L., Feddermann-Demont, N., Giza, C.C., Guskiewicz, K.M., Herring, S., Iverson, G.L., Johnston K.M., Kissick, J., Kutcher, J., Leddy, J.J., Maddocks, D., Makdissi, M., Manley, G.T., McCrea, M., Meehan, W.P., Nagahiro, S., Patricios, J., Putukian, M., Schneider, K.J., Sills A., Tator, C.H., Turner, and M., Vos, VE. (2017) Consensus statement on concussion in sport: the 5th International Conference on Concussion in Sport held in Berlin, October 2016. Br. J. Sports Med. 5 1, 838-847.

2. Tator, C.H. (2013) Concussions and their consequences: current diagnosis, management and prevention. CMAJ 185, 975-979.

3. Waddell, P.A. and Gronwall, D.M. (1984) Sensitivity to light and sound following minor head injury. Acta Neurol. Scand. 69, 270-276.

4. Bohnen, N., Twijnstra, A., Wijnen, G., and Jolles, J. (1991) Tolerance for light and sound of patients with persistent post-concussional symptoms 6 months after mild head injury. J. Neurol. 238, 443-436.

5. Truong, J.Q., Ciuffreda, K.J., Han, M.H., and Suchoff, I.B. (2014) Photosensitivity in mild traumatic brain injury (mTBI): a retrospective analysis. Brain Inj. 28, 1283-1287.

6. Wojcik, S.M. (2014) Predicting mild traumatic brain injury patients at risk of persistent symptoms in the Emergency Department. Brain Inj. $28,422-430$.

7. Chang, T.T., Ciuffreda, K.J., and Kapoor, N. (2007) Critical flicker frequency and related symptoms in mild traumatic brain injury. Brain Inj. 21, 1055-1062.

8. Schrupp, L.E., Ciuffreda, K.J., and Kapoor, N. (2009) Foveal versus eccentric retinal critical flicker frequency in mild traumatic brain injury. Optometry 80, 642-650.

9. Patel, R., Ciuffreda, K.J., Tannen, B., and Kapoor, N. (2011) Elevated coherent motion thresholds in mild traumatic brain injury. Optometry 82, 284-289.

10. Barnett, B.P., and Singman, E.L. (2015) Vision concerns after mild traumatic brain injury. Curr. Treat. Options Neurol. 17, 329.

11. Newsela: Instructional Content Platform. 2017. https://newsela.com

12. Wasserman, E.B., Kerr, Z.Y., Zuckerman, S.L., and Covassin, T. (2016) Epidemiology of sports-related concussions in National Collegiate Athletic Association athletes from 2009-2010 to 2013-2014: Symptom Prevalence, Symptom Resolution Time, and Return-to-Play Time. Am. J. Sports Med. 44, 226-233.

13. Tator, C.H., Davis, H.S., Dufort, P.A., Tartaglia, M.C., Davis, K.D., Ebraheem, A., and Hiploylee, C. (2016) Postconcussion syndrome: demographics and predictors in 221 patients. J. Neurosurg. 125, 12061216.

14. Hiploylee, C., Dufort, P.A., Davis, H.S., Wennberg, R.A, Tartaglia, M.C., Mikulis, D., Hazrati, L., and Tator, C.H. (2017) Longitudinal study of postconcussion syndrome: not everyone recovers. J. Neurotrauma 34, 1511-1523.

15. De Courten-Meyers, G.M. (1999) The human cerebral cortex: gender differences in structure and function. J. Neuropathol. Exp. Neurol. 58, 217-226.

16. Rabinowicz, T., Petetot, J.M., Gartside, P.S., Sheyn, D, Sheyn, T., and De Courten-Myers G.M. (2002) Structure of the cerebral cortex in men and women. J. Neuropathol. Exp. Neurol. 61, 46-57.

17. Broshek, D.K., Kaushik, T., Freeman, J.R., Erlanger, D., Webbe, F., and Barth, J.T. (2005) Sex differences in outcome following sportsrelated concussion. J. Neurosurg. 102, 856-863.

18. Giza, C.C., Hovda, D.A. (2004). The pathophysiology of traumatic brain injury, in: Traumatic Brain Injury in Sports. M. R Lovell, J.T. Barth, and M.W. Collins (eds): Swets \& Zeitlinger: Lisse, The Netherlands, pp. 45-70.

19. Giza, C.C., Hovda, D.A. (2014) The new neurometabolic cascade of concussion. Neurosurgery 75(Suppl 4), S24-S33.

20. Katz, B.J., Digre, K.B. (2016) Diagnosis, pathophysiology, and treatment of photophobia. Surv. Ophthalmol. 61, 466-477. 
21. Shumskaya, E., Andriessen, T.M., Norris, D.G., and Vos, P.E. (2012) Abnormal whole-brain functional networks in homogeneous acute mild traumatic brain injury. Neurology 79, 175-182.

22. Capo-Aponte, J.E., Urosevich, T.G., Temme, L.A., Tarbett, A.K., and Sanghera, N.K. (2012) Visual dysfunctions and symptoms during the subacute stage of blast-induced mild traumatic brain injury. Mil. Med. $177,804-813$

23. Truong JQ, Ciuffreda KJ. (2016) Objective pupillary correlates of photosensitivity in the normal and mild traumatic brain injury populations. Mil. Med. 181, 1382-1390.

24. Doble, J.E., Feinberg, D.L., Rosner, M.S., and Rosner, A.J. (2010) Identification of binocular vision dysfunction (vertical heterophoria) in traumatic brain injury patients and effects of individualized prismatic spectacle lenses in the treatment of postconcussive symptoms: a retrospective analysis. PM R 2, 244-253.

25. Noseda, R., Kainz, V., Jakubowski, M., Gooley, J.J., Saper, C.B., and Digre, K. (2010) A neural mechanism for exacerbation of headache by light. Nat. Neurosci. 13, 239-245.

26. Fimreite, V., Willeford, K.T., and Ciuffreda, K.J. (2016) Effect of chromatic filters on visual performance in individuals with mild traumatic brain injury (mTBI): a pilot study. J. Optom. 9, 231-239.

27. Beasley, I.G., and Davies, L.N. (2013) The effect of spectral filters on reading speed and accuracy following stroke. J. Optom. 6, 134-140.

28. Jackowski, M., Sturr, J., Taub, H., and Turk, M. (1996) Photophobia in patients with traumatic brain injury: uses of light-filtering lenses to enhance contrast sensitivity and reading rate. Neurorehabilitation 6 , 193-201.

29. Yuhas, P.T., Shorter, P.D., McDaniel, C.E., Earley, M.J., and Hartwick, A.T. (2017) Blue and red light-evoked pupil responses in photobic subjects with TBI. Optom. Vision Sci. 94, 108-117.

30. Lynch, J.M., Anderson, M., Benton, B., and Green, S.S., (2015) The gaming of concussions: a unique intervention in postconcussion syndrome. J. Athl. Train. 50, 270-276.
31. Clark, J., Hasselfeld, K., Bigsby, K., and Divine, J. (2017) Colored glasses to mitigate photophobia symptoms posttraumatic brain injury. J. Athl. Train. 52, 725-729.

32. Wilkins, A.J., Baker, A., Amin, D., Smith, S., Bradford, J., Zaiwalla, Z., Besag, F.M.C., Binnie, C.D., and Fish, D. (1999) Treatment of photosensitive epilepsy using coloured glasses. Seizure 8, 444-449.

33. Wilkins, A.J., Patel, R., Adjamian, P., and Evans, B.J. (2002) Tinted spectacles and visually sensitive migraine. Cephalalgia 22, 711-719.

34. Takahashi, Y., Sato, T., Goto, K., Fuljino, M., Fujiwara, T., Yamaga, M., Isono, H., and Kondo, N. (2001) Optical filters inhibiting television-induced photosensitive seizures. Neurology 57, 1767-1773.

35. Blehm, C., Vishnu, S., Khattak, A., Mitra, S., and Yee, R.W. (2005) Computer vision syndrome: a review. Surv. Opthalmol. 50, 253262.

36. Committee on sports-related concussions in youth, Board on children, youth and families. (2014). Sports-Related Concussions in Youth: Improving the Science, Changing the Culture. R. Graham, F.P. Rivara, M.A. Ford, M. and C.M. Spicer (eds). Washington D.C.: National Academies Press.

37. Truong, J.Q., Joshi, N.R., and Ciuffreda, K.J. (2017) Influence of refractive error on pupillary dynamics in the normal and mild traumatic brain injury (mTBI) populations. J. Optom. 11, 93-102.

Address correspondence to: Charles H. Tator, OC, MD, PhD Toronto Western Hospital 399 Bathurst Street, Room 4W-422

Toronto, Ontario M5T 1S8

Canada

E-mail: Charles.Tator@uhn.ca 


\section{Participant Questionnaire}

Please complete this questionnaire after completing the reading tasks. Please note that participation in this survey is completely voluntary.

Study ID:

Date:

Please rate your experience with the following aspects of the non-LCD activity:

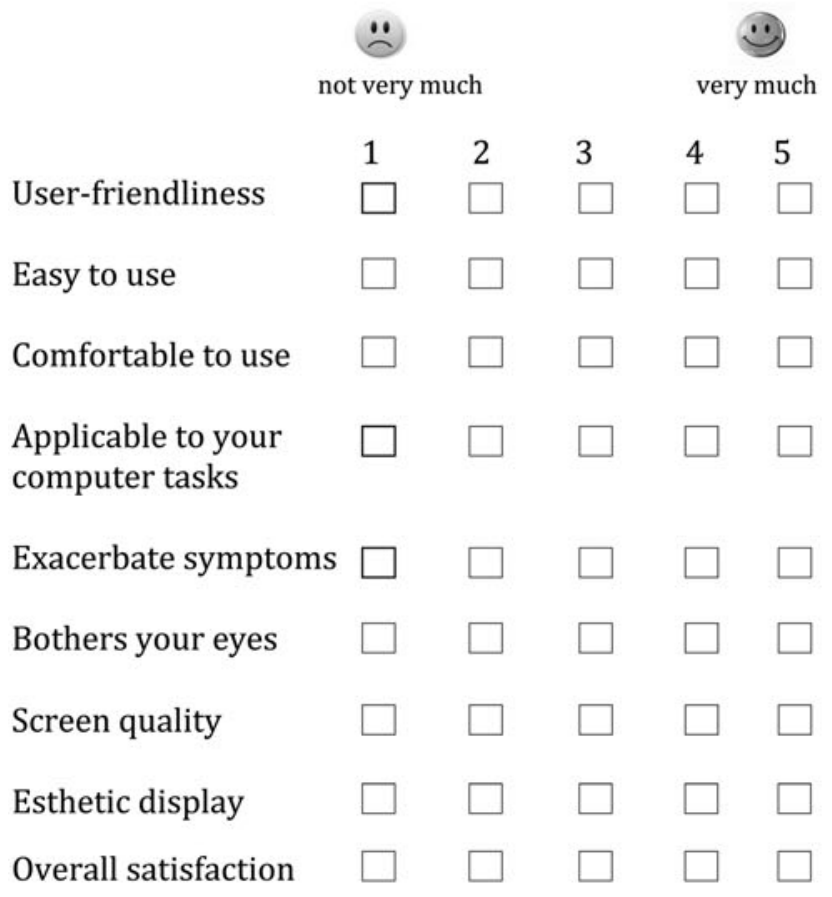

How would you compare your experience with the non-LCD to the LCD computer in regards to the following aspects:

$$
\text { Same Better Worse }
$$

Exacerbate

symptoms

Length of time

reading

Comprehension of text provided

Ease of use

User-friendliness

Comfortable
Which symptom(s) got worse with the non-LCD device, if any?

Which symptom(s) got worse with the LCD-backlit computer, if any?

What aspects of the non-LCD experience did you enjoy?

How can the non-LCD device be improved?

What factors would you consider if you were to buy a non-LCD device for yourself?

Would non-LCD devices be useful for patients with postconcussion syndrome and photosensitivity?

Yes

No

Would non-LCD devices be useful in workplaces and schools for patients with postconcussion syndrome who are returning to work or returning to school?

Yes

No

Would you buy non-LCD device for yourself?

Yes

No

Would you recommend a non-LCD device to others with postconcussion syndrome and

photosensitivity?

Yes

No 